\title{
Diagnostic Value of Sestrin2 in Patients with Obstructive Sleep Apnea
}

\author{
Jiagui Chai, BS, ${ }^{1,2, *}$ Jun Wang, MD, ${ }^{3, *}$ Rong Jiang, BS, ${ }^{1,2}$ Haoyan Wang, PhD, ${ }^{3}$ \\ Huifen Zhai, MD, ${ }^{3}$ Yuanyuan Zheng, MD, Xiaohua Du, PhD, \\ Huilin $\mathrm{He}, \mathrm{MD}^{1}$, Yan Fang, MD, and Shibo Sun, $\mathrm{PhD}^{1}$
}

\begin{abstract}
Background: Obstructive sleep apnea (OSA) is a serious threat to individual health. Diagnosis of OSA is mainly polysomnography (PSG). However, PSG monitoring is costly and time-consuming. At present, increasing studies are exploring new diagnostic methods for OSA. This study aimed to explore the diagnostic role of Sestrin2 in OSA.

Materials and Methods: Sixty-four subjects were recruited in this study. The concentration of plasma Sestrin 2 of all subjects were measured and compared. Spearman's correlation analysis was used to investigate the correlation between plasma Sestrin2 concentration and other factors. Receiver-operating characteristic (ROC) curve was used to investigate the role of Sestrin2 in the diagnosis of OSA, moderate-severe and severe OSA.

Results: Subjects were divided into OSA group $(n=38)$ and control $(n=26)$. Levels of Plasma Sestrin2 were significantly higher in OSA patients than in controls. Sestrin 2 was positively correlated with oxygen reduction index and negatively correlated with mean oxygen saturation and lowest oxygen saturation. The area under ROC curve (AUC) of Sestrin2 for OSA diagnosis was 0.740 [95\% confidence interval (CI), 0.615-0.842], the cutoff value was $1.86 \mathrm{ng} / \mathrm{mL}$, and the sensitivity and specificity were $81.58 \%$ and $61.54 \%$, respectively. The AUC of Sestrin2 for the diagnosis of severe OSA was 0.801 (95\% CI, $0.682-$ 0.890 ), and the cutoff value was $5.21 \mathrm{ng} / \mathrm{mL}$ exhibiting the sensitivity and specificity of $61.90 \%$ and $90.70 \%$, respectively.
\end{abstract}

Conclusion: Setrin2 is a marker for OSA and may be helpful in the diagnosis of OSA.

Keywords: Sestrin2, obstructive sleep apnea, marker, ROC curve, sensitivity, specificity

\section{Introduction}

$\mathrm{O}$ BSTRUCTIVE SLEEP APNEA (OSA) is a serious threat to individual health. ${ }^{1}$ The method for diagnosing OSA is mainly polysomnography (PSG) monitoring. However, PSG monitoring is costly and time-consuming, and it requires patients to have good compliance and professional technicians to analyze the results. ${ }^{2-4}$ At present, the questionnaire plays a role in screening OSA. However, the accuracy of questionnaire in screening OSA is highly controversial. ${ }^{5}$ Accordingly, some inflammation-related factors are used to diagnose OSA, such as interleukin 6 (IL-6), tumor necrosis factor- $\alpha$ (TNF- $\alpha$ ), and C-reactive protein (CRP) ${ }^{6,7}$ Moreover, oxidative stress is closely related to the pathogenesis of OSA. ${ }^{8}$ Oxidative stress factors, such as 8-isoprostaglandin and thioredoxin (TRX), are helpful in the diagnosis of OSA., ${ }^{9,10}$

${ }^{1}$ Department of Respiratory and Critical Care Medicine, First Affiliated Hospital of Kunming Medical University, Kunming, China.

${ }^{2}$ Clinical Medicine, 2015 \& 2017 Grade, Kunming Medical University, Kunming, China.

${ }^{3}$ Department of Respiratory Medicine, Beijing Friendship Hospital, Capital Medical University, Beijing, China.

*These authors contributed equally to this work.

(C) Jiagui Chai et al. 2020; Published by Mary Ann Liebert, Inc. This Open Access article is distributed under the terms of the Creative Commons Attribution Noncommercial License (http://creativecommons.org/licenses/by-nc/4.0/) which permits any noncommercial use, distribution, and reproduction in any medium, provided the original author(s) and the source are cited. 
Table 1. Demographic Characteristics, Sleep Monitoring Parameters, and Blood Measurements of OSA and Control Groups

\begin{tabular}{lccc}
\hline & Control group $(\mathrm{n}=26)$ & OSA group $(\mathrm{n}=38)$ & $\mathrm{P}$ \\
\hline Age (years) & $38.85 \pm 9.16$ & $39.13 \pm 7.77$ & 76.31 \\
Gender (male, \%) & 69.23 & $92.37 \pm 7.62$ & 0.894 \\
Waist to hip ratio (\%) & $88.00 \pm 7.32$ & $28.26 \pm 4.52$ & 0.574 \\
BMI (kg/m) & $26.91 \pm 4.13$ & $36.79 \pm 20.57$ & $0.026^{*}$ \\
AHI (events/hr) & $2.36 \pm 1.19$ & $32.73 \pm 19.15$ & $<0.001^{*}$ \\
ODI (events/hr) & $1.66 \pm 0.94$ & $38.16 \pm 20.37$ & $<0.001^{*}$ \\
Arousal index (events/hr) & $12.56 \pm 7.04$ & $90.22 \pm 2.96$ & $<0.001^{*}$ \\
Mean oxygen saturation (\%) & $93.50 \pm 0.99$ & $79.37 \pm 8.35$ & $<0.001^{*}$ \\
Lowest oxygen saturation (\%) & $90.59 \pm 1.01$ & $19.69 \pm 9.02$ & $<0.001^{*}$ \\
Oxygen saturation <90\% PRTS (\%) & $0.40 \pm 1.42$ & $331.71 \pm 41.60$ & $0.001^{*}$ \\
Total sleep time (min) & $317.40 \pm 40.36$ & $11.12 \pm 3.21$ & 0.178 \\
Stage of REM (\%) & $13.43 \pm 3.52$ & $88.88 \pm 3.21$ & $0.008^{*}$ \\
Stage of NREM (\%) & $86.58 \pm 3.52$ & $1.79 \pm 1.15$ & $0.008^{*}$ \\
Triglycerides (mM) & $1.47 \pm 0.60$ & $4.29 \pm 0.66$ & 0.150 \\
TC (mM) & $4.13 \pm 0.77$ & $2.47 \pm 0.57$ & 0.371 \\
LDL (mM) & $2.31 \pm 0.59$ & $1.05 \pm 0.20$ & 0.287 \\
HDL (mM) & $0.99 \pm 0.20$ & $4.58 \pm 0.58$ & 0.429 \\
Fasting blood glucose (mM) & $4.35 \pm 0.55$ & $12.26 \pm 4.27$ & $<.124$ \\
ESS (scores) & $6.65 \pm 2.56$ & $<.001^{*}$ \\
\hline
\end{tabular}

Values are indicated as the mean \pm standard deviation.

${ }^{a}$ Values are indicated as percentage.

$* P<0.05$, the results are statistically significant.

AHI, apnea/hypopnea index; BMI, body mass index; ESS, Epworth Sleepiness Score; HDL, high-density lipoprotein; LDL, low-density lipoprotein; NREM, nonrapid eye movement; ODI, oxygen desaturation index; OSA, obstructive sleep apnea; PRTS, percentage of recording time spent; REM, rapid eye movement; TC, total cholesterol.

Sestrin2 is an important oxidative stress factor. ${ }^{11}$ Our previous study found that Sestrin2 was involved in the pathogenesis of OSA and was related to the severity of OSA. ${ }^{2}$ Therefore, we speculated that Sestrin2 might be helpful for diagnosis of OSA. The purpose of this study was to explore the role of Sestrin2 in diagnosis of OSA.

\section{Materials and Methods}

\section{Research population}

During the period from June 2016 to January 2019, we recruited 64 subjects who had undergone PSG due to snoring at the First Affiliated Hospital of Kunming Medical

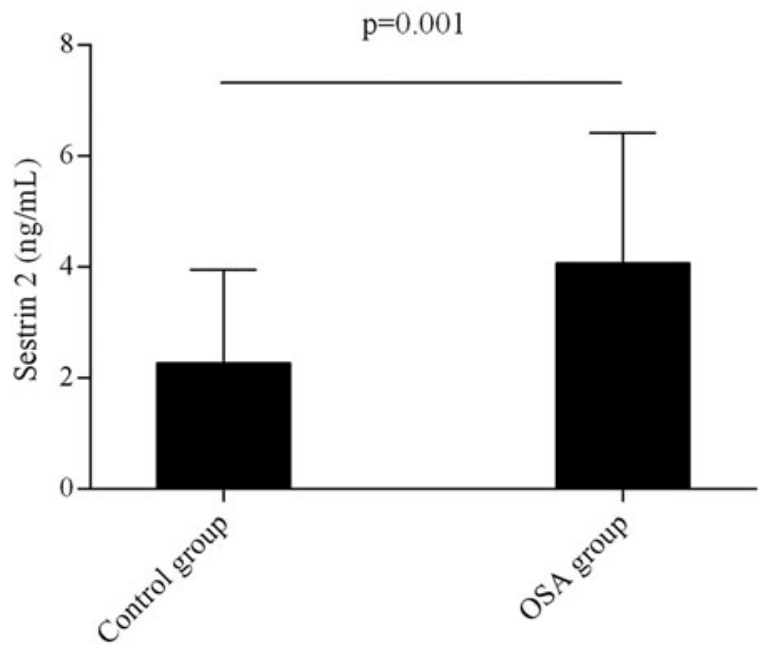

FIG. 1. Comparison in plasma Sestrin2 level between OSA and control groups. OSA, obstructive sleep apnea.
University. Some of the subjects came from Jiang et al., ${ }^{13}$ which was a partly secondary analysis of the subjects from Jiang et al. Before PSG monitoring, all patients underwent systematic physical examination and consultation to obtain their previous health conditions.

The inclusion criteria were subjects aged 18-65 years. The exclusion criteria were patients with lung diseases, such as pneumonia, lung abscess, and so on, heart failure, kidney diseases, cerebrovascular diseases, metabolic diseases such as diabetes, patients with mental disorders, patients with

Table 2. Spearman's Correlation Analysis Between Sestrin2 and Other Factors

\begin{tabular}{lrl}
\hline & \multicolumn{1}{c}{$\mathrm{R}$} & $\mathrm{P}$ \\
\hline Age (years) & 0.036 & 0.832 \\
Waist-to hip-ratio (\%) & 0.064 & 0.703 \\
BMI (kg/m ${ }^{2}$ ) & -0.215 & 0.195 \\
AHI (events/hr) & 0.315 & 0.054 \\
ODI (events/hr) & 0.333 & $0.0041^{*}$ \\
Arousal index (events/hr) & 0.223 & 0.179 \\
Lowest oxygen saturation (\%) & -0.320 & $0.005^{*}$ \\
Mean oxygen saturation (\%) & -0.405 & $0.012^{*}$ \\
Oxygen saturation <90\% PRTS (\%) & 0.054 & 0.748 \\
Total sleep time (min) & -0.055 & 0.744 \\
Stage of REM (\%) & 0.294 & 0.073 \\
Stage of NREM (\%) & -0.294 & 0.073 \\
Triglycerides (mM) & -0.267 & 0.105 \\
TC (mM) & 0.118 & 0.481 \\
LDL (mM) & 0.229 & 0.132 \\
HDL (mM) & 0.476 & $0.002^{*}$ \\
Fasting blood glucose (mM) & -0.184 & 0.270 \\
ESS (scores) & -0.022 & 0.897 \\
Severity of OSA & 0.445 & $0.005 *$ \\
\hline
\end{tabular}

$* P<0.05$, the results are statistically significant. 


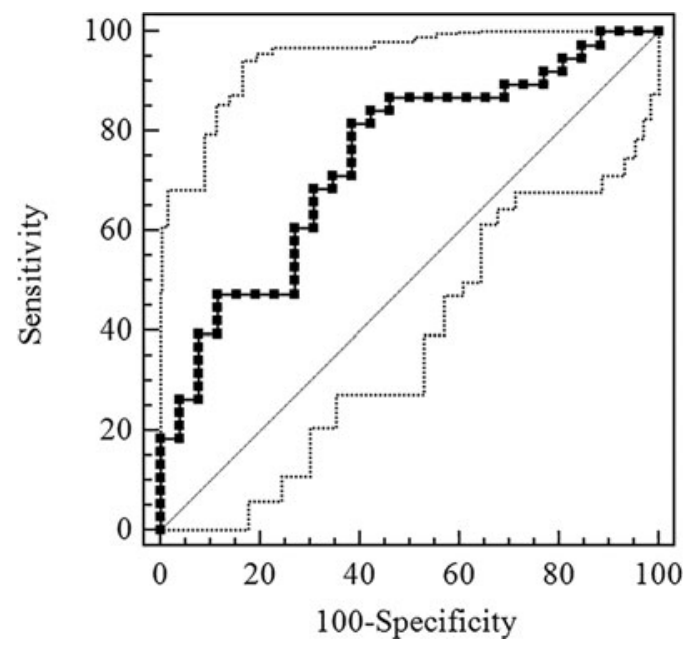

FIG. 2. ROC curve for evaluation of Sestrin2 level of diagnostic effect for OSA. ROC, receiver-operating characteristic.

other sleep disorder-related diseases other than OSA according to history of past illness, pregnancy, and patients with OSA who had received treatment for OSA before this study.

Demographic characteristics, height, body mass index, and waist-hip ratio were measured before PSG. This study was approved by the Ethics Committee of the First Affiliated Hospital, Kunming Medical University.

\section{PSG monitoring}

All subjects were monitored with overnight PSG (Alice 5, America). Apnea was defined as a drop in amplitude of more than $90 \%$ of nasal airflow for at least $10 \mathrm{sec}$. Hypopnea was defined as a drop in airflow amplitude of more than $30 \%$ associated with oxyhemoglobin desaturation of $3 \%$ or more or associated with arousal. ${ }^{14}$ Apnea/hypopnea index (AHI) was obtained after analysis of all PSG records and OSA was stratified according to the American Academy of Sleep Medicine Clinical Practice Guideline. ${ }^{15}$ In addition, central respiratory events were excluded from this study. Normal subjects were defined as AHI $<5$ events/hr, and OSA patients were defined as $\mathrm{AHI} \geq 5$ events/hr.

\section{Plasma test}

On the morning of the day when the PSG monitoring had been completed, the venous blood was taken from all subjects after fasting for more than $8 \mathrm{hrs}$ and centrifuged at $4{ }^{\circ} \mathrm{C}$ for $20 \mathrm{~min}$ (speed $3000 \mathrm{rpm}$ ), and the supernatant was stored in a refrigerator (SANYO, Japan) at $-80^{\circ} \mathrm{C}$ for testing.

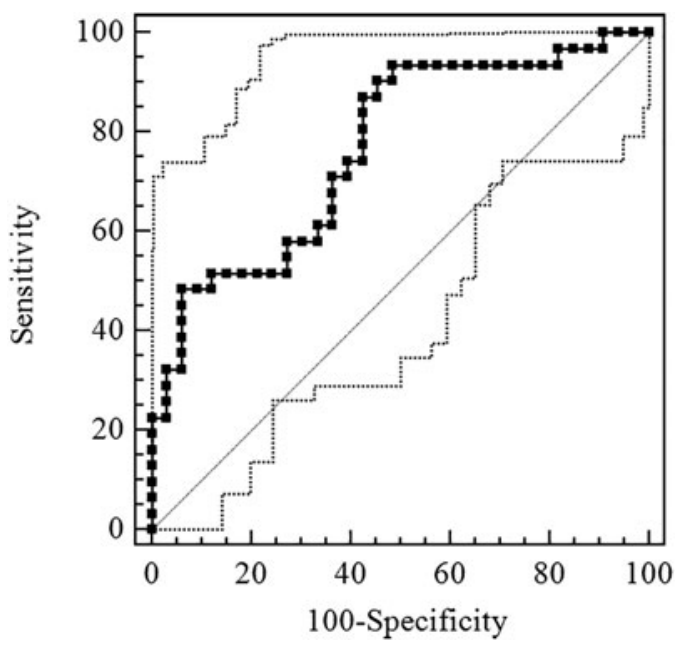

FIG. 3. ROC curve for evaluation of Sestrin2 level of diagnostic effect for moderate-severe OSA.

Sestrin2 was detected with ELISA kit (YAD, China). Other tests, including fasting glucose, triglycerides, total cholesterol, low-density lipoprotein (LDL), and high-density lipoprotein (HDL) were performed by the First Affiliated Hospital, Kunming Medical University.

\section{Statistical analysis}

Data are presented as mean \pm standard deviation. In addition, the single sample Kolmogorov-Smirnov method was used to detect the distribution of data. The data of normal distribution were compared by unpaired $t$-test and that of nonnormal distribution by rank sum test. Spearman's correlation analysis was used to analyze the correlation between Sestrin2 and other parameters. The area under receiver-operating characteristic (ROC) curve (AUC) was used to investigate the diagnostic efficiency of Sestrin2 concentration for OSA, moderate-severe OSA, and severe OSA, respectively. ROC curve drawing and AUC calculation used MedCalc 18.11 (Ostend, Belgium), and other statistical analysis was performed using SPSS 17.0 (Chicago, Illinois).

\section{Results}

Sixty-four subjects were enrolled in this study and were divided into two groups: OSA group $(n=38)$ and control group $(n=26)$. OSA group was divided into three subgroups according to AHI: mild group $(15>\mathrm{AHI} \geq 5)$, moderate group $(30>\mathrm{AHI} \geq 15)$, and severe group $(\mathrm{AHI} \geq 30)$. The results of plasma Sestrin2 and characteristics of all subjects are listed in Table 1.

Table 3. Characteristic of Cutoff Value Concentration for Sestrin2 in Diagnosis of OSA

\begin{tabular}{lcccc}
\hline Sestrin2 & Sensitivity $(95 \%$ CI) & Specificity $(95 \%$ CI) & Positive LR & Negative LR \\
\hline$>0.81 \mathrm{ng} / \mathrm{mL}$ & $100.00(90.70-100.00)$ & $11.54(2.4-30.2)$ & 1.13 & 0.00 \\
$>1.86 \mathrm{ng} / \mathrm{mL}$ & $81.58(65.7-92.3)$ & $61.54(40.6-79.8)$ & 2.12 & 0.30 \\
$>6.74 \mathrm{ng} / \mathrm{mL}$ & $18.42(7.7-34.3)$ & $100.00(86.8-100.0)$ & - & 0.82 \\
\hline
\end{tabular}

$\mathrm{AUC}=0.740(95 \% \mathrm{CI}, 0.615-0.842)$.

AUC, area under receiver-operating characteristic curve; CI, confidence interval; LR, likelihood ratio. 
Table 4. Characteristic of Cutoff Value Concentration for Sestrin2 in Diagnosis of Moderate-SeVere OSA

\begin{tabular}{lcccc}
\hline Sestrin2 & Sensitivity $(95 \%$ CI $)$ & Specificity $(95 \%$ CI) & Positive LR & Negative LR \\
\hline$>0.81 \mathrm{ng} / \mathrm{mL}$ & $100.00(88.8-100.00)$ & $9.09(1.9-24.3)$ & 1.10 & 0.00 \\
$>1.66 \mathrm{ng} / \mathrm{mL}$ & $93.55(78.6-99.2)$ & $51.52(33.5-69.2)$ & 1.93 & 0.13 \\
$>6.74 \mathrm{ng} / \mathrm{mL}$ & $22.58(9.6-41.1)$ & $100.00(89.4-100.0)$ & - & 0.77 \\
\hline
\end{tabular}

$\mathrm{AUC}=0.766(95 \% \mathrm{CI}, 0.644-0.863)$.

The concentration of Sestrin2 in OSA was significantly higher than that in control group (Fig. 1).

Table 2 shows that Sestrin2 was positively correlated with the severity of OSA, HDL and oxygen reduction index, and negatively correlated with mean oxygen saturation and lowest oxygen saturation.

Figure 2 shows the ROC curve of Sestrin2 for OSA diagnosis. The AUC was 0.740 [95\% confidence interval (CI), $0.615-0.842$ ] and the optimal cutoff value was $1.86 \mathrm{ng} / \mathrm{mL}$, exhibiting the sensitivity and specificity of $81.58 \%$ and $61.54 \%$, respectively (Table 3 ). Figure 3 shows the ROC curve of Sestrin 2 for the diagnosis of moderate-severe OSA, the AUC was 0.766 (95\% CI, 0.644-0.863), and the optimal cutoff value was $1.66 \mathrm{ng} / \mathrm{mL}$, exhibiting the sensitivity and specificity of $93.55 \%$ and $51.52 \%$, respectively (Table 4). Figure 4 shows the ROC curve of Sestrin 2 for the diagnosis of severe OSA, the AUC was 0.801 (95\% CI, 0.682-0.890), and the optimal cutoff value was $5.21 \mathrm{ng} / \mathrm{mL}$, exhibiting the sensitivity and specificity of $61.90 \%$ and $90.70 \%$, respectively (Table 5).

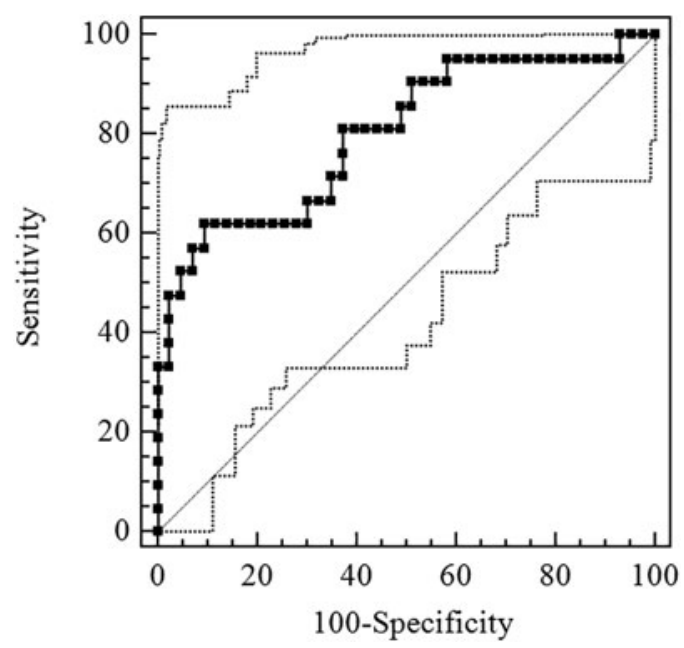

FIG. 4. ROC curve for evaluation of Sestrin2 level of diagnostic effect for severe OSA.

\section{Discussion}

In this study, the concentration of Sestrin 2 in plasma was significantly higher in OSA group than that in control group, suggested that Sestrin2 might differentiate OSA from non-OSA. Moreover, the concentration of Sestrin2 in plasma was positively correlated with the severity of OSA, suggested that OSA severity would be judged by Sestirn2 elevation, which met with the requirements for ideal biomarker. ${ }^{16}$ Further analysis showed that Sestrin2 diagnosed OSA and severe OSA with AUC of 0.740 and 0.801 , respectively, suggested that Sestrin2 was helpful for diagnosis of OSA, especially for severe OSA.

Sestrin2, a member of the Sestrin family, consists of two globular subdomains with similar structure, and is a stress-induced metabolism regulator. ${ }^{11,17}$ The expression of Sestrin2 is mediated by hypoxia or a series of reactions induced by hypoxia. Intermittent hypoxia (IH) is ubiquitous in OSA, ${ }^{18}$ which induces the production of hypoxiainducible factors $1^{19}$ and results the elevating expression of Sestrin $2 .^{20}$ Meanwhile, IH induces strong oxidative stress,${ }^{21}$ leading to excessive accumulation of ROS, which in turn activates antioxidant response elements (AREs). Consequently, AREs induce nuclear erythroid-related factor 2 (Nrf2)-mediated expression of Sestrin2. ${ }^{22}$

Moreover, endoplasmic reticulum (ER) stress and oxidative stress can induce each other. ${ }^{23}$ It may also be the cause of Sestrin2 expression. On the one hand, ER stress induces the production of Sestrin2 through a variety of complex pathways, ${ }^{24}$ and on the other hand, oxidative stress may cause elevating expression of Sestrin 2 by the pathway as described in previous study. ${ }^{22}$ Therefore, hypoxia or/and oxidative stress is an important cause of Sestrin2 increasing in OSA. ${ }^{12}$ In addition, activated Sestrin 2 indirectly regenerates peroxidase (Prx) by promoting the expression of TRX, or removes the Nrf2 inhibitor Kelch-like epichlorohydrinassociated protein 1 (keap1) to activate the antioxidant transcription process, ${ }^{24,25}$ to exert antioxidant effects.

Some explored new ways to diagnose OSA, such as portable monitoring (PM). ${ }^{26,27}$ To some extent, it can make up for the defects of PSG. ${ }^{28}$ However, some suggested that the diversity of PM devices and multiscoring schemes had a significant negative impact on the diagnostic

Table 5. Characteristic of Cutoff Value Concentration for Sestrin2 in Diagnosis of Severe OSA

\begin{tabular}{lcccc}
\hline Sestrin2 & Sensitivity $(95 \%$ CI) & Specificity $(95 \%$ CI) & Positive LR & Negative LR \\
\hline$>0.81 \mathrm{ng} / \mathrm{mL}$ & $100.00(83.9-100.00)$ & $6.98(1.5-19.1)$ & 1.07 & 0.00 \\
$>5.21 \mathrm{ng} / \mathrm{mL}$ & $61.90(38.4-81.9)$ & $90.70(77.90-97.40)$ & 6.65 & 0.42 \\
$>6.74 \mathrm{ng} / \mathrm{mL}$ & $33.3(14.6-57.00)$ & $100.00(91.8-100.0)$ & - & 0.67 \\
\hline
\end{tabular}

$\mathrm{AUC}=0.801(95 \% \mathrm{CI}, 0.682-0.890)$. 
effectiveness of OSA. ${ }^{29,30}$ Moreover, questionnaires are often used to screen OSA. ${ }^{5}$ However, OSA is often accompanied by cognitive deficits, ${ }^{31}$ so that its accuracy is not enough to rely on. Accordingly, some biomarkers with diagnostic accuracy and simple operation were used to diagnose OSA.

Currently, inflammation-related factors, such as IL-6, TNF- $\alpha$, and CRP, are considered as potential biomarkers for the diagnosis of OSA. ${ }^{6,7}$ Fleming et al. ${ }^{32}$ obtained the AUC of diagnosing OSA (moderate-severe) by CRP was 0.73 , and Li et al. ${ }^{10}$ even obtained the AUC of diagnosing OSA by IL-6 was 1.00, which suggested that inflammationrelated factors as biomarkers might be helpful in diagnosing OSA. Oxidative stress is an important pathological feature of OSA. ${ }^{33}$ Guo et al. ${ }^{9}$ found that the TRX, as an oxidative stress factor, was a biomarker of OSA exhibiting the sensitivity and specificity of $91 \%$ and $78 \%$, respectively. In addition, 8-isoprostaglandin, another oxidative stress factor, could also be used as a biomarker to identify OSA. ${ }^{10}$

Our previous study found that Sestrin2 was involved in OSA, and it was positively related to the severity of OSA. ${ }^{13}$ Unfortunately, we did not discuss whether Sestrin2 was helpful for the diagnosis of OSA, which limited its clinical usefulness. On the basis of previous study, we included more subjects and reanalyzed the results, and results showed AUC of Sestrin2 for the diagnosis of moderate-severe OSA and severe OSA was 0.766 and 0.801 , respectively. In view of the above findings, Sestrin 2 may be recommended as a valuable marker for diagnosing OSA, especially for diagnosing severe OSA. Our findings provide a theoretical basis for the clinical application of Sestrin2.

There were some limitations in this study. The sample size of this study was small and it was not conducted randomly. So randomized, multicenter, large-sample studies are needed to confirm the accuracy of Sestrin2 in diagnosing OSA in future.

\section{Conclusions}

Sestrin2 is a marker for OSA diagnosis and may be helpful in the diagnosis of OSA.

\section{Data Availability}

The data used to support the conclusions of the study are included within this article.

\section{Author Disclosure Statement}

No conflicting financial interests exist.

\section{Funding Information}

This work was supported by the National Natural Science Foundation of China (nos. 81560589, 30960450, 81173110, 81402991), the Yunnan Provincial Science and Technology Department [nos. 2019FE001 (-058)], Yunnan Provincial Department of Education (nos. 2017zzx201, 2018JS161) and Teaching and Reform Program of Kunming Medical University (no. 2017-JY-Y040).

\section{References}

1. Sharma N, Lee J, Youssef I, et al. Obesity, cardiovascular disease and sleep disorders: Insights into the rising epidemic. J Sleep Disord Ther 2017;6:260.

2. Jordan AS, McSharry DG, Malhotra A. Adult obstructive sleep apnoea. Lancet 2014;383:736-747.

3. Collop NA, Anderson WM, Boehlecke B, et al. Clinical guidelines for the use of unattended portable monitors in the diagnosis of obstructive sleep apnea in adult patients. Portable Monitoring Task Force of the American Academy of Sleep Medicine. J Clin Sleep Med 2007;3:737-747.

4. De Benedetto M, Garbarino S, Sanna A. Obstructive sleep apnea (OSA): Healthcare and social costs. Med Lav 2017; 108:310-313.

5. Chiu HY, Chen PY, Chuang LP, et al. Diagnostic accuracy of the Berlin questionnaire, STOP-BANG, STOP, and Epworth sleepiness scale in detecting obstructive sleep apnea: A bivariate meta-analysis. Sleep Med Rev 2017;36:57-70.

6. Canto Gde L, Pachêco-Pereira C, Aydinoz S, et al. Biomarkers associated with obstructive sleep apnea: A scoping review. Sleep Med Rev 2015;23:28-45.

7. Gaines J, Vgontzas AN, Fernandez-Mendoza J, et al. Obstructive sleep apnea and the metabolic syndrome: The road to clinically-meaningful phenotyping, improved prognosis, and personalized treatment. Sleep Med Rev 2018;42:211219.

8. Lavie L, Vishnevsky A, Lavie P. Evidence for lipid peroxidation in obstructive sleep apnea. Sleep 2004;27:123128.

9. Guo Q, Wang Y, Li QY, et al. Levels of thioredoxin are related to the severity of obstructive sleep apnea: Based on oxidative stress concept. Sleep Breath 2013;17:311316.

10. Li Y, Chongsuvivatwong V, Geater A, et al. Exhaled breath condensate cytokine level as a diagnostic tool for obstructive sleep apnea syndrome. Sleep Med 2009;10:95-103.

11. Ho A, Cho CS, Namkoong S, et al. Biochemical basis of Sestrin physiological activities. Trends Biochem Sci 2016; 41:621-632.

12. Bai L, Sun C, Zhai H, et al. Investigation of urinary Sestrin2 in patients with obstructive sleep apnea. Lung 2019; 197:123-129.

13. Jiang R, Wang Q, Zhai H, et al. Explorating the involvement of plasma Sestrin2 in obstructive sleep apnea. Can Respir J 2019;2019:2047674.

14. Iber $\mathrm{C}$, Ancoli-Israel $\mathrm{S}$, Chesson $\mathrm{A}$, et al. The AASM Manual for the Scoring of Sleep and Associated Events: Rules, Terminology and Technical Specifications, 1st ed, Westchester, IL: American Academy of Sleep Medicine, 2007.

15. Kapur VK, Auckley DH, Chowdhuri S, et al. Clinical practice guideline for diagnostic testing for adult obstructive sleep apnea: An American Academy of Sleep Medicine Clinical Practice Guideline. J Clin Sleep Med 2017;13:479_ 504.

16. Shih JL, Malhotra A. Could vitamins be helpful to patients with sleep apnea. Chest 2011;139:237-238.

17. Kim H, An S, Ro SH, et al. Janus-faced Sestrin 2 controls ROS and mTOR signalling through two separate functional domains. Nat Commun 2015;6:10025.

18. Levy P, Tamisier R, Minville C, et al. Sleep apnoea syndrome in 2011: Current concepts and future directions. Eur Respir Rev 2011;20:134-146. 
19. Semenza GL. Regulation of oxygen homeostasis by hypoxia-inducible factor 1. Physiology (Bethesda) 2009;24: 97-106.

20. Essler S, Dehne N, Brune B. Role of sestrin2 in peroxide signaling in macrophages. FEBS Lett 2009;583:3531-3535.

21. Wang Y, Chai Y, He X, et al. Intermittent hypoxia simulating obstructive sleep apnea causes pulmonary inflammation and activates the Nrf2/HO-1 pathway. Exp Ther Med 2017;14:3463-3470.

22. Shin BY, Jin SH, Cho IJ, et al. Nrf2-ARE pathway regulates induction of Sestrin-2 expression. Free Radic Biol Med 2012;53:834-841.

23. Cao SS, Kaufman RJ. Endoplasmic reticulum stress and oxidative stress in cell fate decision and human disease. Antioxid Redox Signal 2014;21:396-413.

24. Pasha M, Eid AH, Eid AA, et al. Sestrin2 as a novel biomarker and therapeutic target for various diseases. Oxid Med Cell Longev 2017;2017:3296294.

25. Chen SD, Yang JL, Lin TK, et al. Emerging roles of Sestrins in neurodegenerative diseases: Counteracting oxidative stress and beyond. J Clin Med 2019;8:E1001.

26. de Oliveira ACT, Martinez D, Vasconcelos LFT, et al. Diagnosis of obstructive sleep apnea syndrome and its outcomes with home portable monitoring. Chest 2009;135: 330-336.

27. Erman MK, Stewart D, Einhorn D, et al. Validation of the ApneaLink for the screening of sleep apnea: A novel and simple single-channel recording device. J Clin Sleep Med 2007;3:387-392.

28. Cooksey JA, Balachandran JS. Portable monitoring for the diagnosis of OSA. Chest 2016;149:1074-1081.
29. Krishnaswamy U, Aneja A, Kumar RM, et al. Utility of portable monitoring in the diagnosis of obstructive sleep apnea. J Postgrad Med 2015;61:223-229.

30. Abrahamyan L, Sahakyan Y, Chung S, et al. Diagnostic accuracy of level IV portable sleep monitors versus polysomnography for obstructive sleep apnea: A systematic review and meta-analysis. Sleep Breath 2018;22: 593-611.

31. Olaithe M, Bucks RS, Hillman DR, et al. Cognitive deficits in obstructive sleep apnea: Insights from a meta-review and comparison with deficits observed in COPD, insomnia, and sleep deprivation. Sleep Med Rev 2018;38:39-49.

32. Fleming WE, Ferouz-Colborn A, Samoszuk MK, et al. Blood biomarkers of endocrine, immune, inflammatory, and metabolic systems in obstructive sleep apnea. Clin Biochem 2016;49:854-861.

33. Lavie L. Oxidative stress-A unifying paradigm in obstructive sleep apnea and comorbidities. Prog Cardiovasc Dis 2009;51:303-312.

Address correspondence to: Shibo Sun, PhD

Department of Respiratory and Critical Care Medicine First Affiliated Hospital of Kunming Medical University No. 295, Xichang Road Kunming 650032 Wuhua District

China

E-mail: shibosunky@126.com 